Ebisu Ebisu

Études japonaises Études japonaises

50 | automne-hiver 2013

Création et valeurs dans le Japon moderne

\title{
Le système des ventes d'œuvres d'art réservées aux professionnels au Japon (kōkankai)
}

日本における美術商のためのアートオークション一交換会の活動と 重要性

The Art Auction System for Professionals in Japan (kōkankai)

\section{Cléa Patin}

\section{OpenEdition}

\section{Journals}

Édition électronique

URL : http://journals.openedition.org/ebisu/1190

DOI : 10.4000/ebisu. 1190

ISSN : 2189-1893

Éditeur :

Institut français de recherche sur le Japon (UMIFRE 19 MAEE-CNRS), Maison franco-japonaise

\section{Édition imprimée}

Date de publication : 1 octobre 2013

Pagination : 187-216

ISSN : 1340-3656

\section{Référence électronique}

Cléa Patin, « Le système des ventes d'œuvres d'art réservées aux professionnels au Japon (kōkankai) », Ebisu [En ligne], 50 | automne-hiver 2013, mis en ligne le 21 juin 2014, consulté le 22 avril 2019. URL : http://journals.openedition.org/ebisu/1190 ; DOI : 10.4000/ebisu.1190 


\title{
Le système des ventes d'œuvres d'art réservées aux professionnels au Japon (kōkankai)
}

\author{
Cléa PATIN*
}

À la fin des années 1980, les grands auctioneers - Sotheby's et Christie's en tête - ont dénoncé unanimement la "fermeture " du marché japonais et son "manque de transparence ", considérés comme des freins à leur implantation dans l'archipel. Ce discours sur un marché de l'art opaque, peu bénéfique aux artistes et aux acheteurs, a été relayé par les premières maisons de vente aux enchères japonaises, comme Shinwa Art Auction ou Mainichi Auction, qui ont connu un essor fulgurant au début des années 2000. En effet, ces chantres de la ligne néo-libérale qui appelaient les acteurs japonais du marché de l'art à se soumettre au « libre jeu de la concurrence » et aux « lois du marché », se sont heurtés à un système préexistant de ventes par adjudication, qui fonctionnait de manière autonome et formait, par ses multiples barrières à l'entrée, un obstacle direct à la dérégulation du marché : les kōkankai 交換会 (réunions d'échanges). Organisées par et pour les marchands, elles constituaient - et constituent aujourd'hui encore - un exemple de circulation des biens en cercle quasi-fermé, qui aboutit à une formation artificielle de la valeur des œuvres d'art : les cotes sont fixées dans les limites d'une fourchette définie par ces experts (parmi lesquels le " maître de cérémonie " joue un rôle central), afin d'éviter une envolée trop brusque des cours, qui serait due à des comportements spéculatifs, ou au contraire un tassement trop net, dommageable pour l'ensemble de la

* Université de Tokyo/chercheuse associée à l'Umifre 19 MAEE-CNRS, MFJ. 
profession. En d'autres termes, il s'agit d'une soupape atténuant les aléas du marché. Si très peu d'informations filtrent à l'extérieur sur les codes et les règles qui régissent les kōkankai - ce monde sait bien garder ses secrets -, elles demeurent néanmoins un "facteur clé pour comprendre le marché de l'art $^{1} " \mathrm{au}$ Japon. De fait, leur fonctionnement nous oblige à reconsidérer, a contrario, la valeur sur le plan sociologique d'un marché régulé, opaque, fermé. En quoi le manque de transparence, érigé en système, a-t-il permis de limiter l'envolée des cotes en période d'euphorie et surtout, comment a-t-il contribué à assurer la survie des galeristes, principaux vecteurs de soutien aux artistes, en temps de crise ? Quelles en sont les limites?

Nous nous situons ici dans une perspective sociologique ${ }^{2}-$ et donc relativiste -, dans la mesure où nous considérons que la valeur des biens d'art est le fruit de pratiques culturelles élaborées par des "instances légitimantes" (ici les syndicats de marchands) et le résultat de "circonstances particulières" (les kökankai ont pour but d'atténuer les chocs sur la profession causés par l'évolution parfois chaotique de l'environnement macroéconomique). Grâce à la collaboration de plusieurs galeristes ayant une longue carrière, qui nous ont fait part de leur expérience et de leurs réflexions, nous avons pu entrevoir trois points qui cimentent l'édifice des kökankai : 1) une histoire ancienne, ancrée dans la tradition du commerce de l'art au Japon, 2) des méthodes de vente très originales, 3) et enfin l'existence de financements croisés et de soutiens mutuels.

\section{Une pratique originale et ancienne}

Avant d'aborder précisément le statut des kōkankai au Japon ainsi que les conditions pour en devenir membre, situons brièvement cette pratique au sein de l'ensemble des méthodes de vente aux enchères.

1. Galerie Kotōken 古陶軒, le 24 septembre 2008.

2. Cet article se fonde sur notre thèse de doctorat: Une approche sociologique du marché de l'art au Japon : réseaux d'intermédiation marchands pour la vente, la circulation, la diffusion et la formation de la valeur des auvres d'art, p. 554-573 (thèse sous la direction du Pr. Pierre-Michel Menger, soutenue le 11 septembre 2012 à l'École des hautes études en sciences sociales, au Centre d'études sociologiques et politiques Raymond Aron. Prix Shibusawa-Claudel 2013). 


\section{Des ventes par adjudication au plus offrant mais non publiques}

Si l'on classe aujourd'hui couramment les groupes des kōkankai, de même que les maisons de vente aux enchères, parmi les acteurs du marché secondaire ${ }^{3}$, cette distinction d'origine occidentale ne s'est faite que récemment au Japon ${ }^{4}$. En revanche, le terme kōkankai remonte à l'époque d'Edo, à la fin du XviII siècle. Le mot kōkan signifie littéralement " échange ", tandis que kai désigne la notion de groupe, de réunion. Le mot échange est à prendre ici au sens de commerce, et non simplement de transfert de bien : il s'agit bel et bien de ventes, qui supposent une contrepartie sur le plan monétaire. Autre point sémantique : que ce soit sous la plume des journalistes, des critiques d'art ou des galeristes, l'anglicisme japonais ökushon オークション, qui correspond exactement à notre terme " enchères ", se trouve de plus en plus utilisé en lieu et place du terme traditionnel nyūsatsu 入札 ${ }^{5}$. Il vient du latin auctio, qui désignait les ventes aux enchères dans la Rome antique, qu'il s'agisse de celles des particuliers ou de celles de l'État, des ventes volontaires ou des ventes forcées.

De nos jours, les principales ventes aux enchères en Europe et aux États-Unis conservent de leurs origines romaines la caractéristique d'être publiques (tout individu solvable peut se porter acquéreur) et ascendantes (elles sont effectuées de manière à ce que toute offre d'un prix plus élevé

3. Il s'agit du marché des reventes, sur lequel un bien a été mis au moins une fois en circulation.

4. Nous renvoyons à l'intervention de Fujita Kazuhito 藤田一人 intitulée « La signification du marché de l'art local au Japon " (Domesutikku na Nihon bijutsukai no igi ドメステイックな日本美術界の意義), lors du colloque «Shakai shisutemu 'geijutsu' to sono hen.yō " 社会システム〈芸術〉とその変容 (L'art en tant que système social et ses transformations), Tokyo Metropolitan University 首都大学東京, 2 mars 2013.

5. Le système japonais d'enchères scellées au premier prix (nyüsatsu seido 入札制度), qui se distingue des enchères orales (seriuri 競り売り) par le fait que l'enchérisseur ne reçoit aucun signal de la part des autres participants lorsqu'il remet son offre, serait né à la fin du XVI ${ }^{\mathrm{e}}$ siècle. Toutefois, il ne concerne alors que des transactions privées. Si le bakufu met en garde les magistrats en charge de l'entretien des infrastructures (kobushin bugyō 小普請奉行) contre les ententes collusoires dans la répartition des marchés publics dès 1661, il faut attendre le début de l'ère Meiji pour que le gouvernement adopte officiellement un système d'enchères sous enveloppe cachetée pour les appels d'offres et les contrats publics (concessions, travaux, prestations ou fournitures), avec la loi sur la comptabilité (Kaikei hō 会計法) de 1889 (Kinoshita et al. 2010). 
que celui proposé par un concurrent remporte le bien mis aux enchères ${ }^{6}$ ). Cela suppose concrètement que l'on ait connaissance du prix proposé par ledit concurrent et que l'enchère soit portée à l'oral. Toutefois, la théorie économique englobe bien d'autres modèles de vente aux enchères ${ }^{7}$. Parmi les plus fréquemment utilisés viennent les enchères sous enveloppe cachetée, l'enchère descendante (le prix de départ diminue jusqu'au premier acte d'achat), l'enchère de type all pay (bien que la vente soit remportée par celui qui a fait la meilleure offre, chaque candidat est obligé de payer la somme annoncée), l'enchère inversée, l'enchère au cadran, etc.

Dans le cas des kökankai, il s'agit principalement de ventes sur offres, faites par écrit. De manière générale, le modèle des enchères sous pli cacheté suppose qu'au moment de l'ouverture de toutes les enveloppes on attribue soit le lot au plus offrant (enchères scellées au premier prix), soit au second enchérisseur (enchères philatélistes, ou encore enchères scellées au second prix ${ }^{8}$ ). Les acquéreurs potentiels ignorent donc le prix jusqu'auquel leurs concurrents seraient prêts à monter lorsqu' ils effectuent leur propre proposition. S'il existe au Japon de très nombreuses enchères sous enveloppe cachetée auxquelles tout un chacun peut participer, qu'il s'agisse de vente directe ou sur catalogue, les kökankai ont pour vocation première de faire circuler les œuvres entre marchands. Ainsi, lorsqu'un professionnel acquiert par lot (lors d'une succession notamment) des biens d'art qui ne correspondent pas forcément à sa spécialité, il peut les transférer à un autre marchand dont la clientèle serait potentiellement intéressée. En ce sens, ce sont des ventes entre professionnels - dans le domaine des œuvres d'art, mais aussi dans celui du livre d'occasion - qui ne peuvent être comparées aux ventes destinées au public.

6. Les enchères ascendantes sont appelées couramment " enchères anglaises ", tandis que les enchères descendantes sont nommées « enchères hollandaises ".

7. On doit la première étude conceptuelle majeure des mécanismes d'enchères à Lawrence Friedman (1956), qui cherchait à anticiper les comportements des agents lors de l'enchérissement. En 1961, William Vickrey a également appliqué une théorie novatrice - celle des jeux - aux mécanismes de l'enchère. Il montre que l'acheteur qui suit trop son intuition ou se laisse emporter par son ambition peut être aveuglé par le surenchérissement des concurrents et acquérir le bien ou le service à un prix nettement supérieur à sa valeur initiale (Vickrey 1961).

8. Ces enchères ayant été théorisées par William Vickrey, on les appelle aussi « enchères de Vickrey ». De nos jours, elles sont souvent utilisées lors des transactions sur internet. 


\section{D'Edo à aujourd'hui : la continuation d'une tradition}

Au Japon, l'existence des kökankai est ancienne. Des ventes par adjudication entre marchands d'art ont été organisées à Kyoto dès l'ère Tenmei (1781-1789), au sein de la corporation des marchands d'antiquités de Chine et de Corée (Karamono-ya gumi 唐物屋組) (Tōkyō bijutsu kurabu 1979 : 25-30). On mentionne aussi à Osaka une première vente par adjudication le $25^{\mathrm{e}}$ jour du $2^{\mathrm{e}}$ mois de 1834 (an 5 de l'ère Tenpō), dans le quartier de Fushimichō 伏見町 (Tōkyō bijutsu kurabu 2006 : 1133). Il s'agit alors d'ustensiles de thé ayant appartenu à la famille du grand maître de thé Kobori Enshū 小堀遠州, qui réalisent un chiffre d'affaires total de 9200 kan 貫 (1 380 boisseaux de riz). À Kyoto, une vente d'envergure est attestée le $26^{\mathrm{e}}$ jour du $4^{\mathrm{e}}$ mois de 1870 , sur les biens de la famille du seigneur Matsudaira 松平 de la province d'Izumo. Si nous savons que des " enchères " ont été organisées à Edo dès le début du $\mathrm{XIX}^{\mathrm{e}}$ siècle ${ }^{9}$, notamment dans les quartiers de Nihonbashi et d'Asakusa, les sources restent imprécises, jusqu'à l'organisation de la première grande vente de l'ère Meiji, en 1871. Celle-ci, effectuée sur le patrimoine de la famille du seigneur Sakai du fief de Himeji (Tōkyō bijutsu kurabu 2006 : 1135), inaugure une longue série, suite à la décision du gouvernement, la même année, d'abolir les domaines et de réduire les privilèges et prébendes héréditaires des nobles ${ }^{10}$. L'appauvrissement de la classe des samouraïs et d'une partie des daimyō conduit en effet nombre de familles à se dessaisir de leurs collections.

Les marchands d'œuvres anciennes ont une longue histoire. Autrefois, ils se réunissaient dans des restaurants ou des auberges, où ils exposaient des peintres. Ils mangeaient, buvaient, puis s'achetaient mutuellement des œuvres. C'était une part

9. Sous l'impulsion d'un maître de cérémonie, une trentaine de marchands se réunissaient dans des salles de restaurant ou d'auberge, louées à cet effet, et festoyaient gaiement en cercle avant de passer aux affaires. En cas d'achat, le paiement avait lieu un mois, voire soixante jours après l'adjudication, tandis que l'organisateur prenait une commission de $10 \%$ à $20 \%$.

10. En 1876, le gouvernement impose aux samouraïs et aux anciens chefs de petits domaines une réduction supplémentaire de leurs revenus. Ainsi, un samouraï de haut rang qui bénéficiait d'une rente de 1000 boisseaux de riz avant la Restauration, n'en perçoit plus que 400 en 1868 ; en 1876, il se voit obligé de subsister avec l'équivalent de 150 boisseaux en monnaie (Akamatsu 1968 : 80). 
importante de leur activité. On peut penser que le mécanisme [des kōkankai] a vu le jour au sein des antiquaires - les marchands d'ustensiles de thé existaient à l'époque d'Edo - pendant les ères Bunka [1804-1818] et Bunsei [1818-1830] ${ }^{11}$.

À partir du début de Meiji, les marchands se rassemblent dans des associations puissantes pour peser sur le marché. D’abord spécialisés dans l'art ancien et les ustensiles de la cérémonie du thé, et diversement connus sous le nom de karamonoya 唐物屋 (marchands d'objets chinois), dōguya 道具屋 (marchands d'objets) ou kottōya 骨董屋 (marchands d'antiquités), ils font peu à peu corps sous l'appellation de kobijutsuya 古美術屋 (marchands d'art ancien). Les marchands spécialisés dans la peinture japonaise traditionnelle 日本画 (nihonga), qui s'implantent dans la seconde moitié de Meiji, sont désignés sous le terme nihongashō 日本画商, tandis que les spécialistes de la peinture à l'huile à l'occidentale, apparus dans les années 1920, prennent le nom de yoggashō 洋画商. Ainsi ont été créés le Tokyo Art Club (Tōkyō bijutsu kurabu 東京美術俱楽部) en 1907, dans le prolongement de l'association Ryūchikai 龍池会 fondée en 1879, de même que le Syndicat japonais des marchands de peinture occidentale (Nihon yōgashō kyōdō kumiai 日本洋 画商共同組合), fondé en 1958, suite à l'alliance de plusieurs yōgashō juste avant la Seconde Guerre mondiale. Un autre groupe extrêmement influent, notamment dans la région du Kantō, le Syndicat des marchands d'art de Tokyo (Tōkyō bijutsu-shō kyōdō kumiai 東京美術商協同組合) ${ }^{12}$ a vu le jour en 1949. Étroitement lié au Tōkyō bijutsu kurabu ${ }^{13}$, de par son histoire, son activité et le profil des participants, il compte aujourd'hui environ cinq cents membres et embrasse tous les domaines d'activité " traditionnels "

11. Galerie Kawafune ギャラリー川舟, le 27 novembre 2010.

12. Fondée à partir du Tōkyō bijutsushō shinkō kumiai 東京美術商親交組合 dont la naissance remonte à 1924, cette organisation a par la suite changé quatre fois de noms, devenant successivement Tōkyō bijutsu-shō kumiai 東京美術商組合, Tōkyō bijutsushō shisetsu kumiai 東京美術商施設組合, puis Tōkyō bijutsu-shō shōgyō kyōdō kumiai 東京美術商商業協同組合, avant de trouver son appellation actuelle. Aujourd'hui, elle est souvent désignée sous l'abréviation Tōbi 東美 (http://www.toobi.co.jp/association/ index.html).

13. Si le Tōkyō bijutsu kurabu a pour but de développer des relations d'amitié entre marchands et de stimuler l'échange d'information, le Tōkyō bijutsu-shō kyōdō kumiai se place plus concrètement du côté des échanges monétaires et commerciaux. 
(kobijutsuya, nihongashō et yōgashō ${ }^{14}$ ). Il se fixe des objectifs très élevés en terme de qualité d'expertise et d'éthique dans la pratique de la profession, mais surtout, il continue de jouer un rôle moteur dans l'organisation des ventes par adjudication entre marchands. Il s'agit sans doute de l'archétype de la kōkankai, active dans les domaines décrits par le tableau 1.

Le Syndicat des marchands de Tokyo accorde la priorité aux peintures classiques d'origine chinoise ou japonaise, aux calligraphies, ainsi qu'aux ustensiles utilisés dans le cadre de la cérémonie du thé. Dans l'art postMeiji, il voit surtout s'échanger des œuvres académiques, qu'il s'agisse de tableaux de nihonga ou de yogga. Les tableaux impressionnistes et modernes des artistes occidentaux occupent en son sein une place mineure, mais ne sont pas absents.

Les kōkankai se concentrent sur des artistes japonais pour lesquels il existe déjà un marché. Par rapport à Sotheby's, l'offre est donc très limitée, mais cela permet de fixer les prix assez facilement. On ne trouve dans le lot qu'une poignée d'artistes étrangers : il s'agit pour l'essentiel d'artistes "modernes » japonais, c'est-à-dire actifs à partir de l'ère Meiji ${ }^{15}$.

Ce syndicat organise deux grandes kökankai par an, au printemps et à l'automne. Cependant, plusieurs ventes similaires se trouvent aussi couplées avec les expositions régulières mises en place par le groupe ${ }^{16}$. De même, le Nouveau Syndicat des marchands d'art (Shin bijutsushō kyōdō kumiai 新美術商協同組合), davantage tourné vers l'art moderne et contemporain, organise, outre ses multiples activités sur le marché de l'art, deux ou trois

14. Plus de la moitié des membres sont tournés vers l'art ancien, ce que reflète la nomination de Shimojō Keiichi 下條啓一 (galerie Kobijutsu Shimojō 古美術下條) comme président. Toutefois, les représentants de nihonga, telles les galeries Murakoshi garō 村越画廊, Awazu garō 粟津画廊, Natsume bijutsu-ten 夏目美術店, Sankeidō 三渓堂 ou Seiryūdō bijutsu 青龍堂美術 ont joué un rôle très important dans la hiérarchie du groupe. À travers ces galeries, le Tōbi a certainement contribué à fixer les cotes des artistes de nihonga.

15. Sotheby’s Japan サザビーズジャパン, le 20 juillet 2007.

16. Le Tōkyō bijutsu-shō kyōdō kumiai organise au sein du Tōkyō bijtusu kurabu, souvent en coopération avec ce dernier, plusieurs événements annuels ouverts au public : la triennale de Tōbi (Tōbi tokubetsu-ten 東美特別展), la foire annuelle d'art de Tōbi (Tōbi āto fea 東美アートフェア), la vente directe (Tōbi shōfuda-kai 東美正札会), l'exposition du $\mathrm{XXI}^{\mathrm{e}}$ siècle (Nijū isseiki-ten 21世紀展), la Cérémonie de thé Seibi (Seibi chakai 済美 茶会), etc. 
"grandes rencontres» (taikai 大会) par an, qui rassemblent des marchands venant de tout le Japon. Le Syndicat des marchands de gravures contemporaines du Japon (Nihon gendai hanga-shō kyōdō kumiai 日本現代版画商協 同組合) gère aussi, outre ses réunions mensuelles, un grand rassemblement par an. Dans l'ensemble, ces ventes sont des hauts lieux d'échange de l'information, de resserrement des liens aussi bien amicaux que commerciaux.

TABLEAU 1

Biens mis en vente au sein du Tōkyō bijutsu-shō kyōdō kumiai

\begin{tabular}{|c|c|c|}
\hline Peinture et sculpture & Artisanat d'art & $\begin{array}{l}\text { Industriel décoratif } \\
\text { (artistique par } \\
\text { destination historique) }\end{array}$ \\
\hline $\begin{array}{l}\text { Peinture japonaise } \\
\text { Nihonga } \\
\text { 日本画 }\end{array}$ & $\begin{array}{l}\text { Objets de thé japonais } \\
\text { Nihon chadogu } \\
\text { 日本茶道具 }\end{array}$ & $\begin{array}{l}\text { Objets de lettré } \\
\text { Kogan bunbōgu } \\
\text { 古玩文房具 }\end{array}$ \\
\hline $\begin{array}{l}\text { Estampes japonaises } \\
\text { Nihon hanga } \\
\text { 日本版画 }\end{array}$ & $\begin{array}{l}\text { Céramique japonaise } \\
\text { Nihon tōjiki-hin } \\
\text { 日本陶磁器品 }\end{array}$ & $\begin{array}{l}\text { Objets de } \\
\text { décoration intérieure } \\
\text { Shitsunai sōshoku hin } \\
\text { 室内装飾品 }\end{array}$ \\
\hline $\begin{array}{l}\text { Calligraphies } \\
\text { Nihon sho } \\
\text { 日本書 }\end{array}$ & $\begin{array}{l}\text { Laque japonais } \\
\text { Nihon urushi-hin } \\
\text { 日本漆品 }\end{array}$ & $\begin{array}{l}\text { Textile } \\
\text { Fukushoku } \\
\text { 服飾 }\end{array}$ \\
\hline $\begin{array}{l}\text { Peinture à l'huile japonaise } \\
\text { Nihon abura-e } \\
\text { 日本油絵 }\end{array}$ & $\begin{array}{l}\text { Objets de thé chinois } \\
\text { Chügoku chadōgu } \\
\text { 中国茶道具 }\end{array}$ & \\
\hline $\begin{array}{l}\text { Peinture chinoise } \\
\text { Chügoku-ga } \\
\text { 中国画 }\end{array}$ & $\begin{array}{l}\text { Objets de thé coréens } \\
\text { Chōsen chadogu } \\
\text { 朝鮮茶道具 }\end{array}$ & \\
\hline $\begin{array}{l}\text { Calligraphies chinoises } \\
\text { Chügoku-sho } \\
\text { 中国書 }\end{array}$ & $\begin{array}{l}\text { Céramique chinoise } \\
\text { Chügoku tōjiki-hin } \\
\text { 中国陶磁器品 }\end{array}$ & \\
\hline $\begin{array}{l}\text { Peinture } \\
\text { à l'huile occidentale } \\
\text { Kaigai abura-e } \\
\text { 海外油絵 }\end{array}$ & $\begin{array}{l}\text { Laque chinois } \\
\text { Chügoku urushi-hin } \\
\text { 中国漆品 }\end{array}$ & \\
\hline $\begin{array}{l}\text { Sculptures } \\
\text { Chökoku } \\
\text { 彫刻 }\end{array}$ & & \\
\hline
\end{tabular}


Elles stimulent l'activité des galeristes pendant les mois difficiles. Mais surtout, elles permettent de s'approvisionner à des prix très raisonnables.

Les kōkankai ont pour but de réunir des œuvres, ce qui facilite l'approvisionnement ainsi que l'échange d'informations. Grâce à elles, le marché est stable. Par ailleurs, elles ont aussi leur utilité en cas de vol : la galerie dont un client aurait été victime d'un cambriolage fait état des dommages à ses consœurs. Il devient alors très difficile pour le voleur d'écouler le fruit de ses rapines sur le marché. (...) Fondamentalement, les kökankai permettent de trouver ce que l'on cherche à des prix très raisonnables (à prix de professionnels). En plus, il existe des facilités de paiement. Ainsi, même les mois où l'on n'a pas pu vendre à des collectionneurs particuliers ou à des musées, on donne quand même l'impression d'avoir travaillél ${ }^{17}$.

\section{Estimation des effectifs par domaines de spécialité}

On compte aujourd'hui plus d'une vingtaine de kōkankai à Tokyo et à Osaka, et une cinquantaine sur tout le territoire. Toutes entretiennent leur part de mystère : les règles de fonctionnement interne, les effectifs, et $a$ fortiori les prix d'adjudication et le nom des acquéreurs ne sont pas rendus publics. Comme le suggère le tableau 2, il existe deux types de kökankai. Les kojin-kai 個人会, qui ont lieu à l'initiative d'un organisateur privé et supposent le versement d'une commission au dit organisateur. Toutefois, contrairement à ce que pourrait laisser entendre le mot kojin (individu), celui-ci peut être aussi bien une personne physique que morale (de fait, il s'agit le plus souvent d'une galerie). À l'inverse, les sōgo-kai 相互会 sont gérées de manière entièrement collective. L'ensemble des membres de l'association se partage alors les bénéfices.

Tous les mois, des groupes privés de marchands s' « échangent " donc des œuvres afin de fixer le cours du marché pour les professionnels (oroshiuri shijo 卸売市場). Ce travail régulier d'évaluation des biens d'art, voire de contrôle arbitraire des cotes, puisqu'il s'agit de maintenir les prix à des niveaux acceptables pour les galeristes, tant à la hausse qu'à la baisse, nécessite un niveau d'expertise extrêmement élevé. Cette qualité est exigée pour pouvoir entrer dans le groupe (nous allons bientôt aborder les critères de sélection), mais surtout pour y rester : avant une vente, nulle estimation

17. Nichidō garō 日動画廊, le 2 février 2008 . 
susceptible d'évoquer le prix plancher du vendeur (saitei kyōbai kakaku 最低競売価格) n'est jamais faite. De plus, nous l'avons vu, les participants ne reçoivent aucun signal de la part des autres enchérisseurs. En conséquence, ils ne peuvent s'en remettre qu'à leur propre jugement. Or, un écart trop important de leur(s) prix par rapport à la moyenne du groupe risque de les décrédibiliser aux yeux de leurs confrères, dans le cas de la vente sous pli scellé départagée de manière collective, ou de l'organisateur, dans le cas de la vente par retournement du bol (wan-buse nyūsatsu 椀伏せ入札) ${ }^{18}$.

Proches dans leurs domaines d'expertise, certains syndicats entrent en concurrence directe. Ainsi, dans le domaine de l'art post-Meiji, le Syndicat des marchands d'art de Tokyo (Tōkyō bijutsu-shō kyōdō kumiai), liée au Tokyo Art Club, et le Syndicat japonais des marchands de peinture occidentale (Nihon yōga-shō kyōdō kumiai), inféodé à l'influente galerie Nichidō garō, entrent souvent en conflit lorsqu'il s'agit d'authentifier ou d'expertiser des œuvres. Chacun tente alors de se ménager l'appui des chercheurs. Par exemple, le petit-fils du peintre Umehara Ryūzaburō 梅原龍三郎 (1888-1986), expert reconnu de ses œuvres et détenteur de ses sceaux, se retrouve souvent en position délicate d'arbitre entre les deux. La directrice de la galerie Nichidō, qui cherche à établir un catalogue raisonné définitif du peintre en lieu et place du catalogue existant (incomplet, car essentiellement focalisé sur les peintures à l'huile et les gravures) se heurte souvent aux estimations contradictoires de ses rivaux ${ }^{19}$.

\section{Un marché fermé et hiérarchisé : les barrières à l'entrée}

La population des kōkankai se reconfigure régulièrement, soit que les membres âgés décèdent, soit qu'il y ait de nouveaux entrants - héritiers, successeurs ou novices. Pourtant, des barrières à l'entrée, plus ou moins strictes selon les organisations, régulent le nombre des effectifs. La principale a trait à un système de garantie, qui permet de préserver les intérêts du groupe en cas de faillite de l'un des membres : dans ce cas, le garant (hoshōnin 保証人) se substitue au débiteur pour honorer sa dette. Dans les

18. Nous aborderons la question des modes d'adjudication dans la troisième section. 19. Entretien avec l'arrière-petite-fille du peintre Umehara Ryūzaburō, le 13 novembre 2009. 


\section{TABLEAU 2}

\section{Les principales kōkankai par structure et spécialité}

\begin{tabular}{|c|c|c|c|}
\hline \multicolumn{4}{|c|}{$\begin{array}{l}\text { - INITIATIVE 《INDIVIDUELLE " (kojin-kai 個人会) } \\
\rightarrow \text { l'organisateur de la vente (kaishu 会主) prend une commission }\end{array}$} \\
\hline \multirow{7}{*}{$\begin{array}{l}\text { Art ancien, } \\
\text { nihonga et } y \bar{g} g a\end{array}$} & \multicolumn{2}{|c|}{ Nom } & \multirow{2}{*}{ Eff. } \\
\hline & Shinwa-kai & 親和会 & \\
\hline & Senzoku-kai & 千束会 & 250 \\
\hline & Tsukiji-kai & 築地会 & 230 \\
\hline & Yōka-kai & 八日会 & 200 \\
\hline & Shinbikai & 心美会 & 200 \\
\hline & Shinkōkai & 心交会 & 200 \\
\hline $\begin{array}{l}\text { Gravures, estampes, } \\
\text { artisanat d'art, art } \\
\text { contemporain }\end{array}$ & $\begin{array}{l}\text { Tōkyō gendai bijutsu garō } \\
\text { kaigi }\end{array}$ & 東京現代美術画廊会議 & 12 \\
\hline \multicolumn{4}{|c|}{$\begin{array}{l}\text { - INITIATIVE « COLLECTIVE » (sōgo-kai 相互会) } \\
\rightarrow \text { les membres du syndicat se partagent l'ensemble du bénéfice }\end{array}$} \\
\hline \multirow{7}{*}{$\begin{array}{l}\text { Art ancien, } \\
\text { nihonga et yōga }\end{array}$} & $\begin{array}{l}\text { Tōkyō bijutsu-shō kyōdō } \\
\text { kumiai }\end{array}$ & 東京美術商協同組合 & 500 \\
\hline & Ōsaka gashō sōgo-kai & 大阪画商相互会 & 80 \\
\hline & Nihonga-shō sōgo-kai & 日本画商相互会 & 70 \\
\hline & Nihon yōga-shō kyōdō kumiai & 日本洋画商協同組合 & I \\
\hline & Zenkoku bijutsu-shō sōgo-kai & 全国美術商相互会 & I \\
\hline & $\begin{array}{l}\text { Kyōdō kumiai bijutsu-shō } \\
\text { kōyūkai }\end{array}$ & 協同組合美術商交友会 & I \\
\hline & Kansai bijutsu-shō renmei & 関西美術商連盟 & I \\
\hline \multirow{5}{*}{$\begin{array}{l}\text { Gravures, estampes, } \\
\text { artisanat d'art, art } \\
\text { contemporain }\end{array}$} & $\begin{array}{l}\text { Nihon ukiyoe-shō } \\
\text { kyōdō kumiai }\end{array}$ & 日本浮世絵商協同組合 & 50 \\
\hline & $\begin{array}{l}\text { Nihon gendai hanga-shō } \\
\text { kyōdō kumiai }\end{array}$ & $\begin{array}{l}\text { 日本現代版画商協同 } \\
\text { 組合 }\end{array}$ & 70 \\
\hline & $\begin{array}{l}\text { Shin-bijutsu-shō } \\
\text { kyōdō kumiai }\end{array}$ & 新美術商協同組合 & 60 \\
\hline & $\begin{array}{l}\text { Nihon kenchiku bijutsu kōgei } \\
\text { kyōkai }\end{array}$ & 日本建築美術工芸協会 & $35^{*}$ \\
\hline & $\begin{array}{l}\text { Nihon gyararī nettowāku } \\
\text { kyōkai }\end{array}$ & $\begin{array}{l}\text { 日本ギャラリー・ネット } \\
\text { ワーク協会 }\end{array}$ & 20 \\
\hline
\end{tabular}

Source : Takai (1992: 27) (données réactualisées) 
kökankai les plus ouvertes, un seul parrain suffit. Dans les plus strictes, comme celles organisées par le Nouveau Syndicat des marchands d' $\operatorname{art}^{20}$, il faut être coopté par au moins quatre membres et un administrateur, qui tous recommandent le candidat et se portent caution solidaire. La demande est examinée avec soin par le conseil d'administration qui, dans le cas où il se montrerait favorable, propose à l'ensemble des membres un "vote de confiance ». Une fois accepté par l'ensemble du groupe, le nouvel entrant doit aussi apporter une caution (hoshōkin 保証金), qui lui ouvre le droit d'être membre à part entière. Il peut ensuite être privé de cette qualité en cas de dommage commercial important infligé à un tiers - il tombe alors dans la catégorie "économiquement non fiable " (keizai-teki shinyō fuan 経済的信用不安) -, de plaintes récurrentes de la part de clients ou de poursuites judiciaires.

[Les syndicats] réunissent plusieurs dizaines de marchands d'art, qui cotisent chacun un certain montant en millions de yens pour former une enveloppe globale. Le cœur de leur activité consiste à organiser des ventes aux enchères, réservées aux seuls membres : les kökankai. (...) Tous les nouveaux entrants doivent s'assurer le soutien d'un garant, capable de rassurer les confrères sur sa capacité à payer. Dans certaines $k o ̄ k a n k a i$, on peut avoir à apporter sa caution initiale dans un délai d'un an. Le montant des cotisations varie entre 2,5 millions de yens par personne pour un petit syndicat comme celui des marchands de gravures, et 5 millions pour des groupes au capital plus élevé (de moins en moins nombreux). Vu que la première regroupe une cinquantaine de membres, l'ensemble des cotisations représente au final un montant assez élevé. Ce capital, déposé en banque, nous permet d'emprunter deux à trois fois le montant de la somme ${ }^{21}$.

Selon la sévérité des conditions d'admission, les kōkankai assurent à leurs membres un prestige plus ou moins important, une véritable place dans le milieu.

Le mérite de poser sa candidature dans une kōkankai réside dans le statut que cela procure. On est reconnu socialement comme un vrai marchand. Le nom du groupe a une incidence. Si l'on appartient à une association trop laxiste, cela n'est pas très valorisant : l'on fait face à de fortes disparités en terme d'influence et d'autorité. En

20. Shin bijutsushō kyōdō kumiai dont l'origine remonte à 1978. Voir les règles d'admission sur le site suivant : http://www.gm2000.co.jp/sinbishou.html

21. Shinobazu garō 不忍画廊, le 21 mai 2008. 
revanche, si l'on peut se prévaloir d'un groupe où le parrainage est extrêmement strict et où il faut au moins cinq sceaux de garants, alors c'est un signe de prestige ${ }^{22}$.

De manière générale, les membres des kōkankai obéissent à une hiérarchie très stricte, qui se reflète concrètement dans l'occupation de l'espace. Celle-ci dépend du patrimoine, de l'ascendance, de l'ancienneté, de l'intimité avec les personnes les plus influentes, du degré d'engagement dans les ventes, voire de la participation à des activités bénévoles annexes (préparation des foires d'art, publication d'articles dans des magazines spécialisés, organisation de voyages amicaux, discours sur des thèmes relatifs à l'évolution du marché, organisation de ventes de charité, etc.) destinées à faciliter le bon fonctionnement du groupe. Les personnes les mieux insérées bénéficient de toutes sortes d'avantages en terme de partage des réseaux : on leur présente de nouveaux clients, on les informe des besoins de tel collectionneur, etc.

C'est un marché extrêmement fermé. On ne peut pas prendre de notes, mais tout le monde se regarde, et mémorise chaque mot, chaque geste. J'avais le sentiment que tout ce qui était dit était mémorisé. Ces réunions avaient lieu tous les mois. Les galeries puisent dans leur stock un certain nombre d'œuvres pour les vendre, et en achètent à leur tour. Cela crée donc un marché, en contribuant à fixer les cotes. (...) Il est très difficile de participer à une kökankai... Le cercle des membres est fermé. C'est un monde aussi très masculin ${ }^{23}$.

Dans ces lieux mis en place par les professionnels, les aînés - en âge et en autorité -, de même que les acheteurs les plus assidus, sont dirigés vers les meilleures places. Sur les côtés, des marchands moins avancés dans la hiérarchie, le plus souvent dans leur soixantaine (comme moi), s'activent ostensiblement pour se faire bien voir, dans l'optique d'accéder un jour aux honneurs. Moi, je suis relégué à l'arrière. C'est un monde profondément hiérarchisé, fonctionnant sur le modèle de la promotion à l'ancienneté, un peu comme le salon Nitten. Ensuite, il y a un avantage sur le plan économique, avec la possibilité de se prêter mutuellement de l'argent. Mais il faut sans cesse aller présenter ses salutations aux pontes, accepter leurs invitations, flatter pour réussir. Ces relations doivent être entretenues. En Europe, on dit assez franchement ce que l'on pense, tandis qu'au Japon, l'expression de son opinion se fait de manière plus alambiquée ${ }^{24}$.

22 Ibid.

23. Sotheby's Japan.

24. Galerie Mushanokōji ギャラリー武者小路, le 10 septembre 2008. 
Dans ce monde clos et hiérarchisé, les techniques de vente font l'objet d'un rituel particulier. Parmi les méthodes les plus usitées viennent le système du retournement de bol, la vente sous pli scellé et l'enchère orale avec possibilité de tirage au sort.

\section{Les différentes méthodes d'adjudication}

\section{Le système du « retournement de bol » : wan-buse}

Le système le plus ancien, qui remonte à l'époque d'Edo, est dit du " retournement de bol ». On peut penser que les premières kōkankai ont commencé sur un mode festif, ludique et joyeux, lors des réunions amicales de marchands dans les restaurants et les auberges de Kyoto ou d'Edo. D'où l'usage du «bol » (wan 椀), ou de son couvercle, comme support à l'inscription des prix ${ }^{25}$. Cette coutume a perduré pendant plus d'un siècle et continue de survivre, bien que rare, dans le domaine des rouleaux peints, des manuscrits et des livres anciens, ainsi que pour la peinture nihonga. Le glossaire en ligne de la librairie Seibundō définit ainsi la méthode :

"Il s'agit de l'une des méthodes d'adjudication dans le domaine des livres anciens ; au lieu d'inscrire un prix sur un morceau de papier, on écrit le montant à l'intérieur d'un bol que l'on fait glisser jusqu'à l'organisateur de la vente - le nakaza 中座 -, chargé de retrouver le meilleur enchérisseur. Sur l'extérieur du bol, en laque noir, est inscrit le nom de chaque participant ; par contraste, l'intérieur du bol est en laque vermillon. Cette méthode n'est presque plus utilisée depuis les années $1950^{26}$. ”

Elle est pourtant réapparue dans les années 2000 au sein du Marché aux livres anciens du quartier de Kanda à Tokyo (Tōkyō kosho kaikan 東京古書会館). De manière un peu fortuite, des vétérans de l'Association des libraires de livres anciens de Tokyo (Tōkyō koten-kai 東京古典会) ont en effet redécouvert dans un vieux débarras une trentaine de bols et d'écritoires utilisés autrefois dans les ventes selon la méthode du wan-buse. Le

25. Du côté des libraires, il semblerait que cette pratique soit plus récente : elle serait apparue au début de l'ère Taishō, au sein de l'Association des libraires de livres anciens (Koten-kai 古典会).

26. http://www33.ocn.ne.jp/-seibundo/glossary/wa.html 
directeur de la librairie Seishindō 誠心堂 nous en a rapporté un saisissant témoignage (Hashiguchi 2010). Il nous explique ainsi que toutes les places - des coussins carrés (zabuton 座布団) utilisés pour s'asseoir sur les tatami sont attribuées à l'avance en fonction de la hiérarchie. Une trentaine de participants se font face, tandis que le nakaza préside l'auditoire sur le côté. Les bols sont distribués à l'entrée. L'intérieur, en laque rouge, fait ressortir l'encre noire utilisée pour inscrire le montant. Sous le socle, enduit de laque rouge, se trouve écrit le nom du libraire. Devant chaque coussin est aussi placé une écritoire (suzuribako 硯箱) contenant de l'encre, un encrier en pierre, un pinceau et un linge humide.

Lorsque la vente commence, le nakaza présente d'abord l'objet succinctement, avant de le faire circuler de mains en mains. Les acheteurs potentiels doivent alors effectuer leurs choix très rapidement, d'où la nécessité d'un très haut degré d'expertise. Une fois l'objet revenu au centre, les personnes intéressées inscrivent au fond de leur bol le prix auquel elles sont prêtes à l'acquérir. Elles ne peuvent proposer qu'un seul montant (contrairement au système des ventes sur offre écrites sur papier). Chacun n’a donc qu'une seule chance : il faut bien connaître les cours du marché. Pour maintenir le secret, elles « retournent » leur bol (fuseru 伏せる), puis le lancent vers celui qui dirige et arbitre la vente. Les bols doivent glisser, tout près du sol et arriver pratiquement dans la main du nakaza. La précision du geste est requise à la fois pour faire preuve d'élégance, pour ne pas abîmer le matériel, et pour éviter l'humiliation qui consisterait à révéler son offre aux confrères. Le nakaza rassemble alors les bols, les empile et les trie, avant de révéler à haute voix celui qui remporte le bien. Normalement, il s'agit du plus offrant, mais le nakaza a toute latitude pour imposer son jugement lorsqu'il considère que les cours sont anormalement élevés, ou qu'il y a eu une erreur flagrante d'appréciation. À l'inverse, il peut inciter les participants à ne pas inscrire un montant trop bas, lors de la présentation de l'œuvre.

Après chaque enchère, un préposé (keieiin 経営員) note le résultat dans un registre (yamachō 山帳) prévu à cet effet. Dans le cas où le bien n'aurait pas atteint le prix de réserve du vendeur (tome 止め), il lui est restitué. Une vente qui n’a pas eu lieu est désignée sous le terme « $b \bar{o}$ »ボー (bâton), sans doute parce qu'on l'indique sur le registre d'un simple trait. Une fois sa décision consignée, le nakaza renvoie les bols à leurs propriétaires en les faisant glisser de la même manière, avec souplesse et précision. Les participants se 


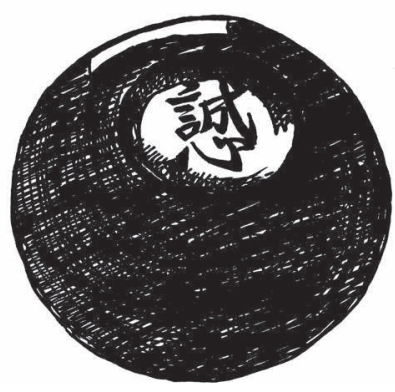

Le bol

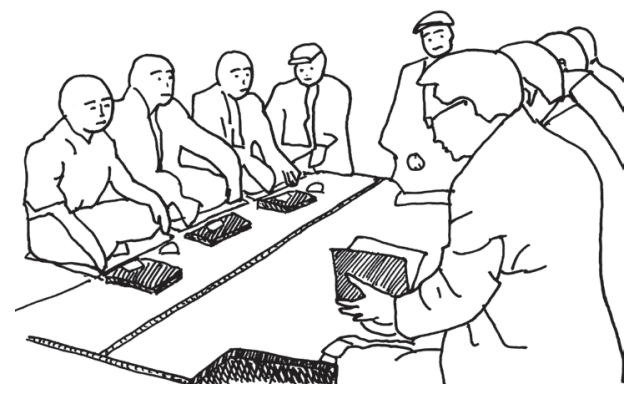

La phase d'estimation

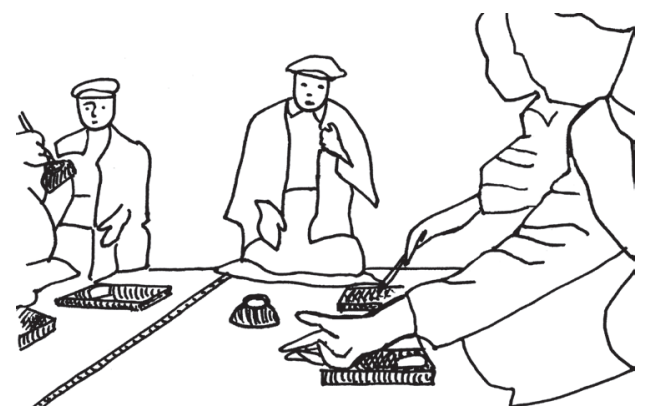

Le lancement du bol

Dessins de l'auteure,

d'après Hashiguchi (2010 : 1-2) 
saisissent alors de leur linge humide et effacent les prix. Ils se préparent pour la vente suivante. Quand la kōkankai se termine, la personne en charge du registre prépare les factures et calcule le montant global de la vente (nuki ヌキ). Les acheteurs peuvent aller voir leurs acquisitions (hikiawase 引き 合わせ) avant de payer et de rentrer chez eux. Une fois un bien acquis, ils ne peuvent en aucun cas le rendre ou réclamer un remboursement, quelle que soit la nature de leur grief. L'autorité absolue du nakaza ne souffre aucune réclamation. Cependant, le paiement peut avoir lieu de manière différée, au bout d'une semaine, voire au bout d'un mois. Les personnes concernées émettent alors une reconnaissance de dette (kinken 金券).

Dans le monde des antiquités et de la peinture nihonga, le système est très similaire. Ici aussi, le nakaza, fort de son expérience, peut arbitrer pour préserver les cours, de manière unilatérale. Il peut suggérer que les prix proposés sont trop faibles, anticipant un effondrement du marché, ou au contraire qu'ils sont trop élevés, auquel cas la vente est annulée. Même si on attend de lui qu'il reste impartial, il peut parfois abuser de son autorité. L'une des personnes que nous avons interviewées souligne ainsi que «si un ami à lui fait partie des participants, il peut user de son influence pour qu'on lui vende ; ce n'est ni un marché transparent, ni un marché juste ${ }^{27}$ ». Par rapport au monde des libraires, quelques éléments different cependant. Tout d'abord, ce n'est pas le plus offrant qui remporte l'enchère, mais celui qui se situe juste au-dessous, afin d'éviter une envolée suspecte des cours. Par ailleurs, il peut arriver que l'on jette deux fois le wan : une première fois pour fixer un prix plafond (les personnes qui n'acceptent pas ce seuil retirent alors leur bol du jeu), et une seconde pour déterminer celui qui gagne le droit d'acheter l'œuvre. Enfin, les participants prennent leur temps lors de l'estimation des œuvres. Voici le témoignage d'un vétéran des kōkankai:

J'utilisais ce genre de bol [il montre le couvercle creux d'un bol à soupe] - peut-être un peu plus gros. On commence par coller un morceau de papier à l'intérieur, sur lequel on inscrit le numéro de la boutique (les antiquaires sont nombreux), puis on fait tourner les biens. Ça prend du temps. Les personnes qui désirent tel ou tel objet écrivent au fond de leur bol le prix auquel elles seraient prêtes à l'acquérir, puis le retournent et le font glisser sur les tatamis en direction du nakaza. Ce dernier les ramasse et les trie. À la fin, il ne garde que le deuxième meilleur enchérisseur

27. Galerie Mushanokōji. 
et annonce son nom. Il ne prend jamais le plus offrant, pour éviter les comportements spéculatifs. En effet, si l'on retenait le prix plafond, une personne pourrait imposer à trois millions de yens un bien qui n'en vaudrait pas plus d'un, et forcer ses confrères à entériner cette hausse subite des cours. De même, les erreurs grossières dans l'estimation des prix sont supprimées (il peut arriver que quelqu'un se trompe et estime à dix millions ce que ses confrères évaluent à un million !). D'où la nécessité de ne proposer qu'un seul prix. Cependant, une personne qui désire vraiment un bien peut ajouter, en direction du nakaza, "je m'en remets à vous ". Ce dernier lui accorde alors une attention particulière. Pour peu qu'il s'agisse d'un très bon acheteur, ou d'une personne envers qui l'on est reconnaissant, il peut le faire passer devant le deuxième meilleur offrant. Par exemple, si la personne propose 520000 yens et que le deuxième enchérisseur se situe à 500000 yens, c'est elle qui l'emporte. Personne n'est en mesure de vérifier : le maître de cérémonie ne montre rien. Les œuvres continuent de circuler, les unes après les autres. Encore une fois, ça prend du temps. Les participants sont triés sur le volet, parce qu'il faut une grande confiance en soi quant à l'estimation des prix. Aujourd'hui, les nombreux novices qui s'installent sur le marché préfèrent le système des enchères à l'occidentale, plus facile. Le nombre de personnes capables de pratiquer le " retournement du bol " diminue. Ce système fonctionnait bien il y a encore dix ou quinze ans, mais il est en perte de vitesse. Peut-être se maintient-il encore au sein des marchands spécialisés dans les ustensiles de thé ${ }^{28}$.

\section{La vente sous pli scellé}

Pratiqué lors des expositions des artistes vivants organisées par les syndicats, qu'il s'agisse de peintres de nihonga ou de yōga, ce type de vente suppose le dépôt de bulletins secrets dans des urnes, un peu à la manière d'un vote. Le 26 mai 2007, lors de la $7^{\mathrm{e}}$ exposition "Créer à partir de la tradition, $\mathrm{xxI}^{\mathrm{e}}$ siècle $^{29} "$, co-organisée par le Tokyo Art Club et le Syndicat des marchands d'art de Tokyo, nous avons ainsi assisté à une vente à bulletin secret. Naturellement, les prix espérés n'étaient pas indiqués sous les tableaux. À l'entrée du bâtiment s'alignaient des boîtes en bois portant chacune le nom d'un artiste. Les marchands - et eux seuls - étaient habilités à glisser un papier dans la fente, sur lequel ils inscrivaient ce qu'ils pensaient être le prix du marché (shijō kakaku 市場価格), ainsi que le montant auquel ils souhaitaient acquérir l'œuvre, s’ils étaient intéressés. Les bulletins étaient

28. Galerie Kawafune.

29. «Dai nana kai dentō kara no sōzō nijū isseiki-ten » 第7回 伝統からの創造 21 世紀展. 
ensuite lus et départagés de manière collective. Contrairement au wan-buse, dans la plupart des ventes sur offre, on peut proposer plusieurs prix, par ordre décroissant : par exemple, trois quand les œuvres se situent au-dessus de 10000 yens, quatre au-dessus de 100000 yens. La possibilité de choisir parmi ces montants laisse aux marchands une certaine latitude dans la fixation des cotes, favorisant plus la moyenne que le plafond.

\section{L'enchère orale ascendante avec tirage au sort}

Méthode la plus proche du système occidental des enchères " à l'anglaise ", ce type de vente est le plus prisé de nos jours. La kōkankai commence alors de manière habituelle : l'organisateur présente, dans un bref discours, les règles de fonctionnement, la liste des biens mis en vente - dont disposent déjà les participants, puisqu'elle leur a été envoyée un mois à l'avance - et l'évolution générale des cours. Les œuvres sont apportées chacune leur tour et déposées sur une estrade devant les participants. L'organisateur annonce le titre de l'œuvre, le nom de l'artiste et, s'il y a lieu, les changements de propriétaires. Ensuite, l'adjudication se fait oralement. Les participants «montent les uns sur les autres » avec des prix de plus en plus élevés, jusqu’à ce que plus personne ne souhaite surenchérir. Comme le résume un galeriste spécialisé dans la céramique ancienne :

On se rassemble entre marchands, entre confrères, jusqu'à trente ou quarante personnes. $\mathrm{Si}$ je veux vendre une œuvre, celle-ci est portée aux enchères. Un peu comme sur le marché aux poissons de Tsukiji ${ }^{30}$.

De prime abord, le processus apparaît donc classique. Pourtant, il peut arriver que plusieurs marchands farouchement déterminés à acquérir l'œuvre risquent, par leur enfièvrement, de faire monter les cours à des niveaux «déraisonnables " (futekisetsu 不適切). Dans ce cas, l'organisateur peut exiger que l'on cesse d'enchérir et que l'on tire au sort, pour un montant déterminé à l'avance. Autrement dit, le nakaza a tout latitude pour rompre l'envolée de l'enchère quand il la juge trop passionnée - un fait inimaginable au sein des maisons de vente occidentales.

30. Galerie Kotōken. 
Si cette méthode profite aux marchands, qui contrôlent les prix et thésaurisent l'information, on peut se demander quelles sont leurs conséquences pour les artistes et les collectionneurs. Du côté des artistes, notamment des jeunes peintres de nihonga, force est de constater que certains peuvent être injustement favorisés au détriment d'autres. Leur cote peut être surévaluée ou sous-évaluée en fonction des attentes des marchands les plus influents, qui imposent leur avis à leurs confrères et acquièrent ainsi un poids déterminant dans la poursuite des expositions publiques dans les salons. Or, les pontes, qui sont aussi les marchands les plus âgés, tendent à sélectionner les disciples des maîtres qu'ils connaissent bien. Cela conduit immanquablement à une reproduction des styles et des thèmes (par exemple, les classiques paysages kachō fügetsu 花鳥風月), à un manque de fraîcheur et de renouvellement au niveau de l'inspiration. Le système rigide de reproduction des carrières en sort consolidé, tandis que les artistes les plus innovants se voient relégués à la périphérie, voire définitivement écartés. Toutefois, en comparaison des risques d'envolée ou d'effondrement des cotes qui se font jour dans les ventes publiques organisées par les nouvelles maisons de vente à l'occidentale, il n'est pas sûr que le système le plus transparent soit forcément le plus bénéfique aux artistes sur le long terme.

Du côté des collectionneurs, l'absence totale de transparence au niveau des transactions et des prix, qui protège l'intérêt des marchands, peut à juste titre susciter la suspicion. En l'absence d'éléments comparatifs, quand les biens n'ont pas été portés en vente publique, il est impossible d'estimer les revenus du vendeur, ses marges ou ses bénéfices. Aucune information ne filtre à l'extérieur sur le chiffre d'affaires retiré des kōkankai, par rapport à celui retiré du marché. Or, malgré l'intervention du nakaza pour enrayer les comportements spéculatifs ou les enfièvrements, l'expérience - certes très éphémère - de la «bulle » de la fin des années 1980 a montré qu'en période d'emballement du marché les prix peuvent tout de même être multipliés par dix, vingt, cent. Enfin, même si une œuvre boudée par les collectionneurs n'atteint jamais un sommet de prix en kōkankai, elle y trouve tout de même un débouché artificiel, ce qui crée une sorte de marché parallèle coupé de la demande réelle, haut lieu de circulation des invendus (fuyō zaiko kōkan no ba 不要在庫交換の場).

En général, on apporte dans les kökankai les pièces que l'on possède depuis longtemps, mais que l'on ne parvient pas à écouler. Il est évident que l'on ne peut deviner 
si elles vont intéresser les autres galeristes, mais on préfère les vendre quitte à essuyer une légère perte. Le mois suivant, on revaudra ça à la personne qui nous aura aidé cette fois-ci ${ }^{31}$.

$\mathrm{Au}$ fond, il s'agit d'une sorte d'association de secours mutuel. On s'entraide. Ce qu'un galeriste ne peut pas vendre, un autre le lui achète, pour le soutenir. C'est l'idée de base. Évidemment, c'est un peu spécial. À partir de maintenant, comment va évoluer le système? C'est une question qui taraude toutes les galeries japonaises ${ }^{32}$.

Une question difficile, d'autant plus que si la solidarité entre marchands constitue le pilier des kökankai, non seulement pour se débarrasser des pièces difficiles à vendre, mais surtout pour se financer mutuellement, le système présente aujourd'hui des failles majeures, tant aux yeux du fisc qui n’apprécie guère l'opacité des transactions, que pour les marchands euxmêmes : l'augmentation des effectifs et la baisse de la qualité des œuvres en circulation compromettent dangereusement la viabilité du système.

\section{Un marché hautement solidaire, mais en perte de vitesse}

Concrètement, la solidarité entre marchands peut prendre la forme d'une compensation pécuniaire pour les perdants lors de la vente avec tirage au sort (voir supra), mais surtout d'un crédit mutuel à partir d'un fonds commun.

\section{Financements et soutiens mutuels}

Que les différentes méthodes d'adjudication entre marchands privilégient en priorité le maintien de cours stables, cela peut se comprendre. Il est cependant une ancienne coutume qui ne manque pas d'étonner les observateurs extérieurs au monde de l'art : lors des tirages au sort effectués à la demande du nakaza, le (ou les) perdant(s) reçoivent une compensation (shügi 祝儀), entièrement à la charge de l'acquéreur, qui peut atteindre, selon le montant de l'œuvre, entre 100000 et 300000 yens. Ces pratiques

31. Galerie Shinobazu.

32. Sotheby's Japan. 
seraient nées dans le milieu des antiquaires au début de l'ère Meiji. Elles perdureraient dans les ventes du Tōbi, mais également dans d'autres groupes plus récents comme la Shinwa-kai, qui a donné naissance à Shinwa Auction (Takai 1992 : 30). Selon un témoin :

Il existe un système compensatoire pour les personnes qui n'ont pas pu acheter l'œuvre. Avant de tirer au sort, on décide collectivement que le montant de la compensation sera par exemple de 10000 yens par personne, puis on tire à la courte paille pour savoir qui emporte l'œuvre. Si dix personnes tirent au sort, alors neuf d'entre elles reçoivent 10000 yens, et le coût de la peinture, qui devait s'élever initialement à 5 millions de yens, atteindra 5,1 millions [90 000 yens de compensation, plus 10000 yens de commission pour la kökankai]. Ce mécanisme vaut pour les œuvres les plus chères (pour les autres, nul besoin de tirer au sort). Toutefois, il tend à disparaître aujourd'hui. Il avait surtout cours dans le monde de la peinture nihonga, mais celui-ci est en train de s'effondrer ${ }^{33}$.

Cependant, cette compensation constitue sans doute un détail au regard de l'importance, véritablement centrale, du système de crédit mutuel. En effet, une galerie qui vendrait une toile est en droit de recevoir son paiement le jour même, presque toujours en liquide (une fois soustraite la commission de $10 \%$ à $15 \%$ due au nakaza, quand il s'agit d'une kojin-kai). Elle peut donc lever des liquidités très rapidement, ce qui constitue un secours non négligeable dans un milieu où l'écoulement des œuvres se fait par à-coups, à un rythme lent et instable, au gré de la conjoncture. L'acquéreur, par contre, bénéficie d'un délai d'un à deux mois - voire de six mois - pour payer. L'ensemble de la kōkankai lui avance donc l'argent. En cas de défaillance, il se voit secourir par son (ou ses) garant(s). Autrement dit, pour peu qu'il ait déjà un client en tête pour l'œuvre, il est en mesure de dégager un bénéfice immédiat, sans intérêt à payer. Ce système de crédit peut être mis à profit même dans le cas où deux galeries se seraient entendues à l'avance sur la vente d'une œuvre. De fait, pour obliger les galeristes à participer aux ventes et s'entraider, un système coercitif d'amende existe aussi.

Il s'agit de transactions commerciales, qui donnent lieu à un échange monétaire. On est parfois gagnant, parfois perdant (il arrive que l'on soit confronté à une baisse des prix). Ainsi, si je vends un tableau pour 100000 yens, on me versera dès le lendemain

33. Galerie Shinobazu. 
la somme de 97000 yens (3\% sont retenus par le syndicat comme commission), en puisant dans le fonds commun. Le capital des marchands tourne. Heureusement d'ailleurs, parce qu'on investirait toutes nos liquidités dans les ouvres d'art et la gestion quotidienne deviendrait impossible. Or, on ne peut vivre de tableaux et d'air pur. Nous avons créé ce système de syndicats avec un fonds commun pour faire en sorte que, lorsque l'un d'entre nous a besoin de liquidités (pour payer son loyer, pour régler les frais d'essence, bref, pour assurer sa subsistance), il puisse vendre via l'association. Sans elle, l'argent ne bougerait pas. Car on ne vend pas tous les mois ${ }^{34}$.

Le vendeur fait tout de suite parvenir l'œuvre à l'acheteur, mais ce dernier bénéficie d'un délai d'un mois pour payer. Or un mois, c'est un facteur non négligeable quand on fait des affaires. Par exemple, si une ouvre coûte cinq millions de yens et que l'on n'a qu'un seul million en liquidités, il est tout de même possible de l'acheter pour le compte d'un client à condition de passer par une kökankai. On paiera le mois suivant. Ce système est vraiment propre au Japon. Grâce à lui, le marché s'est élargi, parce qu'il permettait aux marchands de subsister. (...) Un aspect difficile de notre métier réside dans le fait que le commerce des œuvres d'art se fait à un rythme extrêmement lent. Dans ma galerie, si un client vient acheter une œuvre une fois par semaine, ou même une fois par mois, je suis content. Cela signifie que nous autres galeristes sommes souvent à la limite de la banqueroute. Un mois sans aucune vente nous fait perdre de l'argent, puisqu'il nous faut toujours débourser les frais de fonctionnement. Mettons que ce mois-ci j'aie à payer trois millions de yens. Je n'ai pas d'autre issue que de passer par les enchères de marchands, pour obtenir des liquidités de manière immédiate et éviter d'avoir à brader mes œuvres. Ce système était sans doute l'un des plus efficaces au monde ${ }^{35}$.

C'est pratique. Quand on a besoin d'argent, on peut vendre en grande quantité. Ensuite, on reçoit un paiement au bout d'un mois quand on vend, mais on bénéficie d'un délai de trois à six mois quand on achète. Si les transactions ont lieu au moment du grand rassemblement annuel, on peut négocier de ne payer qu'au bout de six mois. Enfin, toute galerie membre s'engage à investir une certaine somme dans les ventes de l'association - mettons 3 millions de yens par an. En dessous, elle aurait à payer une amende ou serait obligée de se retirer. Cela permet à l'association de s'assurer un certain chiffre d'affaires pour perdurer ${ }^{36}$.

Hauts lieux de la solidarité entre marchands, qui profitent du caractère strictement privé des ventes pour contrôler les cotes, les kōkankai se

34. Idem.

35. Galerie Kotōken.

36. Marchand d'art, le 21 avril 2008. 
heurtent cependant à plusieurs écueils : problèmes d'évasion fiscale, augmentation inconsidérée des effectifs, manque de débouchés extérieurs, etc. Les zones grises des kōkankai menacent à terme leur survie.

\section{Les limites d'un tel marché}

Tout d'abord, si les financements croisés permettent certes à nombre de galeries de subsister, le fisc juge avec sévérité ce qu’il considère comme des «tours de passe-passe ». En effet, vendre dans une kökankai peut permettre de déguiser un déficit ou au contraire, de surestimer des pertes, pour permettre une forme d'évasion fiscale. En 1991, un grand nombre de galeries se sont ainsi vu infliger des contrôles et des redressements de la part de leur centre des impôts pour l'année fiscale précédente. Il leur aurait été reproché des déclarations sous évaluées (kashō shinkoku 過小申告) au regard du volume des transactions, notamment entre confrères (Takai 1992 : 37).

Mais les marchands ne sont pas les seuls concernés. Certains clients peu scrupuleux peuvent aussi espérer jouer sur les zones grises qui entourent la vente discrète en kökankai pour frauder le fisc, ce que ne permettrait pas une excellente mise aux enchères au vu et au su de tous chez les grands auctioneers.

Au Japon, les gens ressentent une certaine honte à vendre les tableaux qui décorent leur maison. Cependant, vu les conditions actuelles de l'habitat, certains sont bien obligés de le faire. Dans des ventes publiques, ils pourraient certainement les écouler rapidement et à des prix élevés, mais passer par une galerie peut aussi avoir son intérêt. Par exemple, le vendeur peut espérer faire un profit sans s'acquitter de l'impôt. Dans une maison de vente aux enchères à l'occidentale, pour un bénéfice autour d'un milliard de yens, plus de la moitié risque de partir en taxes ; certains préfèrent alors confier leur bien à un marchand japonais, capable de l'écouler de manière souterraine, quitte à n'en retirer que 700 millions ${ }^{37}$.

Si les kōkankai ont pu constituer l'un des pans d'une économie souterraine échappant au contrôle du ministère des Finances, rappelons toutefois que ce phénomène n'apparaît pas propre au Japon : selon plusieurs

37. Galerie Mushanokōji. 
économistes, les données relatives aux enchères publiques ne constituent en Europe et aux États-Unis que le quart tout au plus des échanges sur le marché de l'art (Gramp 1989 : 15-16 ; Rouget et alii 1991 : 42). Comme le souligne aussi Raymonde Moulin, "une partie des transactions s'effectue dans la clandestinité, et les phénomènes inquantifiables ou invisibles l'emportent sur les données apparentes et mesurables. Il existe, du fait de l'origine des œuvres et de l'argent, une économie souterraine dont l'importance est difficile à évaluer" (Moulin 2003 : 11).

Les kōkankai, qui ont très bien fonctionné jusqu'à la fin des années 1980, ont subi dans les années 1990, après l'éclatement de la bulle spéculative, des attaques multiples : les assauts du fisc, la grogne des collectionneurs, les violentes critiques de journalistes spécialisés ${ }^{38}$, la concurrence des nouvelles maisons de vente aux enchères, et les doutes émanant des galeristes eux-mêmes. De toute part, la pression pour plus d'ouverture et de transparence se faisait plus forte. Ensuite, marché très solidaire dans la réussite, il se trouvait tout aussi interconnecté et interdépendant dans l'échec : les faillites de certains membres se sont répercutées sur tous les autres, via un effet domino. Pour faire face aux conséquences dramatiques de la récession (selon plusieurs interviewés, $70 \%$ des galeries auraient disparu à la suite de l'éclatement de la bulle), les marchands ont cherché à élargir la base des cotisations : dans de nombreux syndicats, ils se sont montrés moins rigoureux dans les conditions d'admission, en augmentant les effectifs de manière inconsidérée. Dans un cas bien précis, on est ainsi passé d'une vingtaine de membres à plusieurs centaines. L'activité de la kōkankai s'en est trouvée dynamisée, puisque le nombre des ventes par mois a augmenté, mais son fonctionnement s'est détérioré et la qualité des œuvres mises en vente a considérablement baissé ${ }^{39}$. Faute de hiérarchie stricte et d'obéissance absolue à l'autorité du nakaza, ce type de regroupement ne semble donc pas viable. L'un des interviewés dénonce même un nivellement de l'expertise - qui est pourtant le pilier des kökankai-sur le plus bas dénominateur commun.

38. On pense notamment à Takai (1992), ainsi qu'à Itoi (2001).

39. Galerie Shinobazu. 
L'Association des marchands de gravures, c'est moi qui l'ai fondée. Mais maintenant, je n'en suis pas satisfait. Même parmi les marchands, peu de gens comprennent les qualités d'une bonne gravure. Beaucoup ne saisissent même pas la différence entre une reproduction, une simple impression et une œuvre originale créée par un artiste! Tout ce qui compte, c'est de faire monter les prix. Certes, le marché s'est élargi, mais cela ne signifie pas pour autant que les meilleures œuvres aient pu s'imposer dans le monde ${ }^{40}$.

Le principal problème des kōkankai relève de la difficulté à trouver un débouché extérieur au milieu. Si elles peuvent survivre en cercle fermé, elles s'affaiblissent à terme si les œuvres ne trouvent pas d'acheteur.

Si les œuvres ne sortent pas de ce milieu de professionnels pour aller à la rencontre de la demande, alors on évolue en cercle fermé, et ce n'est pas bon ${ }^{41}$.

Même en travaillant au sein des kōkankai, il faut au bout du compte trouver un utilisateur final. Sinon, l'association perd de son dynamisme. Or, il suffit que l'un des membres s'assombrisse pour que tous les autres fassent grise mine. C'est ce qui se passe aujourd'hui ${ }^{42}$.

Ce système fonctionne même trop bien : nous ne pouvons plus nous en passer, au risque de ne plus être en phase avec notre époque. Aujourd'hui, les œuvres proposées n'ont plus de débouchés en dehors des kōkankai. Sans elles, nous serions obligés de mettre la clé sous la porte. Nombre d'entre nous ne parviennent à subsister qu'en vendant des œuvres en cercle fermé. On achète dans une kökankai pour revendre dans une autre, et réciproquement (ce type de passe-passe est monnaie courante). Seulement voilà, en cours de route, nous avons oublié de vendre aux clients, ce qui s'avère extrêmement problématique ${ }^{43}$.

Les kōkankai ont d'autant moins de débouchés à l'extérieur qu'elles se révèlent incapables d'absorber l'art contemporain. De fait, elles se sont bâties jusqu'à la fin des années 1980 en réponse à une forte demande pour l'art figuratif, qu'il s'agisse des peintures yōga et nihonga, des tableaux impressionnistes et post-impressionnistes, ou des œuvres modernes d'avant-guerre.

40. Marchand d'art.

41. Galerie Nichidō.

42. Galerie Shinobazu.

43. Galerie Kotōken. 
Elles prenaient le relais du système académique de formation des carrières (salons Inten, Nitten, etc.) et des cercles de peintres. Artistes, marchands, experts (membres des jurys) et collectionneurs partageaient alors des intérêts mutuels. Or, à l'heure où un changement s'est opéré à l'échelle mondiale au bénéfice de l'art contemporain, elles se trouvent distancées, témoins d'une autre époque.

Aujourd'hui, le principal problème des kōkankai réside dans leur incapacité à absorber l'art contemporain. Elles en restent à l'art moderne. Les jeunes galeries dynamiques (Koyama, Koyanagi, etc.), ont été obligées de fuir le marché de l'art japonais pour apprendre des galeries étrangères et retrouver une forme de compétition, de stimulation mutuelle. Certes, l'art moderne demeure important en termes de volume et de prix des transactions, mais à ce rythme, la situation pourrait s'inverser ${ }^{44}$.

Fondées sur des pratiques solidement enracinées de financement et de solidarité mutuels, les ventes aux enchères réservées aux seuls membres des syndicats de marchands continuent de constituer un système propre au Japon. Bien que largement décriées, elles ont longtemps assuré la survie du système traditionnel de lancement et de consécration des artistes, via leurs interactions avec les chefs de file des cercles de peintres et les experts des jurys des grands salons. Dans les périodes d'envolée du marché, elles ont servi de soupape pour l'approvisionner en ouvres, permettant aux marchands de réaliser des marges importantes. En période de récession, elles ont garanti un débouché stable et régulier aux galeries dans l'embarras, qu'il s'agisse de crise conjoncturelle ou de la difficulté structurelle à trouver des acquéreurs. Elles ont facilité les relations entre concurrents potentiels, stimulant l'élargissement des réseaux et le partage d'informations sur la valeur des œuvres, l'évolution des cotes, les besoins des acheteurs. Bref, elles ont permis - et continuent de permettre - à de nombreuses galeries de subsister. Pourtant, de par leur fermeture, leur manque de transparence (les transactions, cachées au monde extérieur, échappent non seulement aux collectionneurs, mais parfois au fisc) et leurs difficultés d'adaptation (elles se révèlent notamment incapables d'absorber l'art contemporain), elles constituent aussi un frein à la modernisation du marché. Concurrencées de front par les auctioneers et par les maisons de vente aux enchères domestiques, elles

44. Idem. 
traversent une crise profonde. Si l'augmentation des effectifs des syndicats a momentanément retardé l'implosion du système en engendrant une hausse du volume des transactions, elle a aussi entraîné une baisse de la qualité des œuvres échangées, un nivellement par le bas du niveau d'expertise, un relâchement des liens entre les membres et une incertitude plus forte sur leur santé économique. Fonctionnant de plus en plus en vase clos, les kōkankai s'affaiblissent à terme sans débouché extérieur. Or, en leur sein, tous sont solidaires, pour le meilleur et pour le pire. Aujourd'hui, les galeries japonaises sont donc à l'affût : du dénouement de cette crise dépendra la survie et la restructuration de tout un pan du marché.

\section{Bibliographie indicative}

AKAMATSU Paul, 1968

Meiji-1868. Révolution et contre révolution au Japon, Paris, Calmann-Lévy.

ASHENFELTER Orley, 1989

"How Auctions Work for Wine and Art ", Journal of Economic Perspectives, $3: 23-36$.

DE MARCHI Neil, 2004

«Auctioning Paintings in Late Seventeenth-Century London: Rules,

Segmentation and Prices in an Emergent Market », in GINSBURGH Victor A. (ed.), Economics of Art and Culture, Amsterdam, Elsevier, pp. 97-128.
DURET-ROBERT François, 1991

Marchands d'art et faiseurs d'or, Paris, Belfond/Connaissance des Arts.

FUJII Kazuo 藤井一雄, 1991

Ōkushon monogatari オークション物語 (Une histoire des ventes aux enchères), Tokyo, Kōdansha 講談社.

GRAMP William D., 1989

Pricing the Priceless: Art, Artists, and Economics, New York, Basic Books.

HASEGAWA Jin 長谷川仁, 1964 Yōgashō 洋画商 (Les marchands de peinture occidentale), Tokyo, Nichidō garō shuppan 日動画廊出版. 
HASHIGUCHI Kōnosuke

橋口侯之介, 2010

«Mukashi no shijō : wan-buse no

saigen » 昔の市場——椀伏せの再現

(Le marché autrefois : reconstitution

de la pratique du wan-buse). En ligne :

www.mmjp.or.jp/seishindo/essay/

eesay-wahon07.pdf

HERBERT John, 1990

Inside Christie's, New York, St Martins

Press.

\section{HERMANN Frank, 1984}

Sotheby's: Portrait of an Auction House,

New York, Norton \& Co.

FRIEDMAN Lawrence, 1956

"A Competitive Bidding Strategy ",

Operations Research, 4 : 104-112.

HOOG Michel

\& HOOG Emmanuel, 1991

Le marché de l'art, Paris, PUF.

ITOI Kei 糸井恵, 2001

Kieta meiga o sagashite 消えた名画を

探して (À la recherche des chefs-d'œuvre disparus), Tokyo, Jiji tsūshin-sha

時事通信社.

KINOSHITA Seiya 木下誠也，

SATŌ Naoyoshi 佐藤直良，

MATSUMOTO Naoya 松本直也,

ASHIDA Yoshinori 芦田義則, 2010

« Kaikeihō ni okeru kōkyō kōji nyūsatsu

seido no rekishi-teki kōsatsu »

会計法における公共工事入札制度の

歴史的考察 (Réflexion historique sur

le système d'adjudication pour les

travaux publics dans le cadre de la loi sur la comptabilité),

Doboku gakkai ronbun shū F4 (Kensetsu

manejimento) tokushūgō 土木学会論文集

F4(建設マネジメント)特集号，

66 (1) : 169-180.

MOULIN Raymonde, 1992

L'artiste, l'institution et le marché, Paris,

Flammarion, coll. « Champs».

MOULIN Raymonde, 2003

Le marché de l'art : mondialisation

et nouvelles technologies, Paris,

Flammarion, coll. « Champs».

Nihon yōgashō kyōdō kumiai

日本洋画商協同組合, 1985

Nihon yōga-shō shi 日本洋画商史

(Histoire des marchands de peinture

occidentale au Japon), Tokyo, Bijutsu

shuppan-sha 美術出版社.

\section{PATIN Cléa, 2012}

Une approche sociologique du marché de l'art au Japon : réseaux d'intermédiation marchands pour la vente, la circulation, la diffusion et la formation de la valeur des cuvres $d$ 'art, thèse de doctorat sous la direction du Pr. Pierre-Michel Menger, soutenue le 11 septembre 2012 à l'École des hautes études en sciences sociales, au Centre d'études sociologiques et politiques Raymond Aron.

\section{ROUGET Bernard,} SAGOT-DUVAUROUX Dominique, PFLIEGER Sylvie, 1991

Le marché de l'art contemporain en France, Paris, La Documentation française. 
SEGI Shin.ichi 瀬木慎一, 1998

Meiga no nedan. Mō hitotsu no Nihon

bijutsu-shi 名画の值段——もう一つの

日本美術史 (Le prix des chefs-d'œuvre : une autre histoire de l'art japonais),

Tokyo, Shinchōsha 新潮社.

\section{SMITH Charles W., 1990}

Auctions: The Social Construction of Value, Berkeley, University of California Press.

TAKAI Hideyuki 高井秀行, 1992

Āto māketto no uragawa :

bijutsuhin o kashikoku kau tame ni アートマーケットの裏側——美術品を 賢く買うために (Les coulisses du marché de l'art : pour acheter intelligement des œuvres d'art), Tokyo, Mainichi shinbun 毎日新聞.

TAKI Teizō 瀧悌三, 2000

Nihon no yōga-kai nanajū nen :

gaka to gashō no monogatari 日本の

洋画界七十年一画家と画商の物語

(Le milieu de l'art japonais de style occidental depuis soixante-dix ans : histoire des relations entre artistes et marchands), Tokyo, Nikkei jigyō shuppansha

日経事業出版社.

\section{Tōkyō bijutsu kurabu}

\section{hyaku-nen shi hensan iin-kai}

東京美術俱楽部百年史編纂委員会, 2006

Bijutsu-shō no hyakunen. Tōkyō bijutsu kurabu hyaku-nen shi 美術商の百年 一一東京美術俱楽部百年史 (Un siècle de commerce d'art : à l'occasion du centenaire du Tōkyō Art Club), Tokyo, Tōkyō bijutsu kurabu/Tōkyō bijutsu-shō kyōdō kumiai

東京美術俱楽部·東京美術商協同組合.

\section{Tōkyō bijutsu kurabu}

東京美術俱楽部, 1979

Tōkyō bijutsu shijō shi 東京美術市場史

(Histoire du marché de l'art tokyoïte),

Tokyo, Tōkyō bijutsu kurabu.

VICKREY William, 1961

"Counterspeculation, Auctions, and Competitive Sealed Tenders ", Journal of Finance, March, 16 (1) : 8-37.

YAMAMOTO Masako 山本真砂子, 2010 Karamono-ya kara bijutsu-shōe. Kyōto ni okeru bijutsu shijō o chūshin ni 唐物屋から美術商へ——京都における 美術市場を中心に (Des karamono-ya aux marchands d'art : au cœur du marché de l'art de Kyoto), Kōyō shobō 晃洋書房. 